Proceedings of the XXIII Conference on Applied Crystallography, Krynica Zdrój, Poland, September 20-24, 2015

\title{
Elastic-Plastic Transition in MBE-Grown GaSb Semiconducting Crystal Examined by Nanoindentation
}

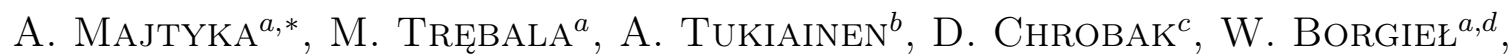 \\ J. RÄISÄNEN ${ }^{e}$ AND R. NOWAK ${ }^{a}$ \\ ${ }^{a}$ Nordic Hysitron Laboratory, Department of Materials Science and Engineering, Aalto University, \\ 00076 Aalto, Finland \\ ${ }^{b}$ Optoelectronics Research Centre, Tampere University of Technology, 33101 Tampere, Finland \\ ${ }^{c}$ Institute of Materials Science, University of Silesia, 75 Pułku Piechoty 1, 45-500 Chorzów, Poland \\ ${ }^{d}$ Institute of Physics, University of Silesia, 75 Pułku Piechoty 1, 45-500 Chorzów, Poland \\ ${ }^{e}$ Department of Physics, University of Helsinki, P.O. Box 43, FI-00014, Helsinki, Finland
}

\begin{abstract}
The present paper concerns the elastic-plastic nanodeformation of Te-doped GaSb crystals grown by molecular beam epitaxy on the $n$-type of GaSb substrate. The conventional analysis of nanoindentation data obtained with sharp triangular (Berkovich) and spherical tip revealed the elastic modulus $(E=83.07 \pm 1.78 \mathrm{GPa})$, hardness $(H=5.19 \pm 0.25 \mathrm{GPa})$ and "true hardness" $\left(H_{T}=5.73 \pm 0.04 \mathrm{GPa}\right)$. The registered pop-in event which indicates the elastic-plastic transition in GaSb crystal points towards the corresponding yield strength $\left(\sigma_{Y}=3.8 \pm 0.1 \mathrm{GPa}\right)$. The origin of incipient plasticity in GaSb crystal is discussed in terms of elastic-plastic deformation energy concept.
\end{abstract}

DOI: 10.12693/APhysPolA.130.1131

PACS/topics: 81.40.Jj, 62.20.F-, 81.15.Hi, 81.05.Ea

\section{Introduction}

The GaSb low band-gap crystal, has attracted considerable interest among the III-V compound semiconductors due to its potential applications for infrared detectors (the Schottky barrier diodes) [1], infrared LEDs [2], thermophotovoltaic generators [3], or spintronics devices [4]. It has turned out to be important candidate for applications in tunnel field-effect transistor, due to its inter-band tunneling capability exhibited when an energy barrier is absent [5]. With the recent development of nanomaterials, the engineers become increasingly aware that present applications of semiconductors require satisfactory mechanical characteristics in addition to already well-established electronic and optical properties. Suffice it to mention as an example the ultra-thin cantilever with resonant frequencies modified by an accurate adjustment of its elastic constants, which is currently used as an efficient nanoelectromechanical sensor [6]. While the nanomechanical properties of numerous semiconductors had already been studied (see e.g., Refs. [7-9]), there is lack of systematic data about mechanical behavior of the important GaSb crystal. Consequently, our research addresses the nanoindentation probing of this interesting material. Our focus is the elastic and plastic behavior of nanodeformed $\mathrm{GaSb}$ thin film fabricated by molecular beam epitaxy (MBE).

\section{Materials and methods}

The GaSb thin film was grown by MBE using the V80H-10 system equipped with standard chamber designed for deposition of group-III elements with the

\footnotetext{
* corresponding author; e-mail: anna.majtyka@aalto.fi
}

growth rate $1 \mu \mathrm{m} / \mathrm{h}$ and at temperature $500^{\circ} \mathrm{C}$. In this study we investigated the $500 \mathrm{~nm}$ thick layer of $n$ type GaSb:Te with $10^{16} \mathrm{~cm}^{-3}$ doping levels deposited on $500 \mathrm{~nm}$ thick $n$-type GaSb(100) substrate. Antimony was provided from a Valved cracker source and tellurium dopants were introduced by a special GaTe source.

The nanoindentation experiments were performed using Hysitron Triboindenter TI 950 equipped with either the sharp Berkovich (nominal radius $R=100 \mathrm{~nm}$ ) or spherical $(R=1 \mu \mathrm{m})$ diamond tip. The measurements were carried out in a load-control-mode with maximum indentation load $P_{\max }$ varied from 100 to $5000 \mu \mathrm{N}$. The selected load time $(180 \mathrm{~s})$ allowing us to observe the discontinuities (pop-in events) on a loading part of the loaddisplacement $(P-h)$ curve.

\section{Results and discussion}

The typical $P-h$ curves registered during the indentation of the sharp-tip are smooth and repeatable, which indicates the homogeneity of our sample (Fig. 1a).

Based on the obtained results we determined the conventional Mayer hardness $(H)$ and Young modulus $(E)$ (see Fig. 1b) of the examined material, according to the Oliver and Pharr method [10]:

$$
\begin{aligned}
& H=\frac{P}{A}, \quad \frac{1-\nu^{2}}{E}=\frac{1}{E_{\text {eff }}}-\frac{1-\nu_{i}^{2}}{E_{i}}, \\
& E_{\text {eff }}=\frac{\sqrt{\pi} S}{2 \beta \sqrt{A}},
\end{aligned}
$$

where $P, A, S, E_{i}, E_{\text {eff }}, \nu, \nu_{i}$, and $\beta$ define the indentation load, contact area, unloading stiffness, indenter elastic modulus, effective Young modulus, material Poisson ratio, the Poisson ratio of diamond tip and constant dependent on the Berkovich tip geometry - 1.034 [11], respectively. The calculated values of $H=5.19 \pm 0.25 \mathrm{GPa}$ and 
$E=83.07 \pm 1.78 \mathrm{GPa}$ (see Fig. 1b) are consistent with the earlier data that were obtained by means of microindentation equipment [12].
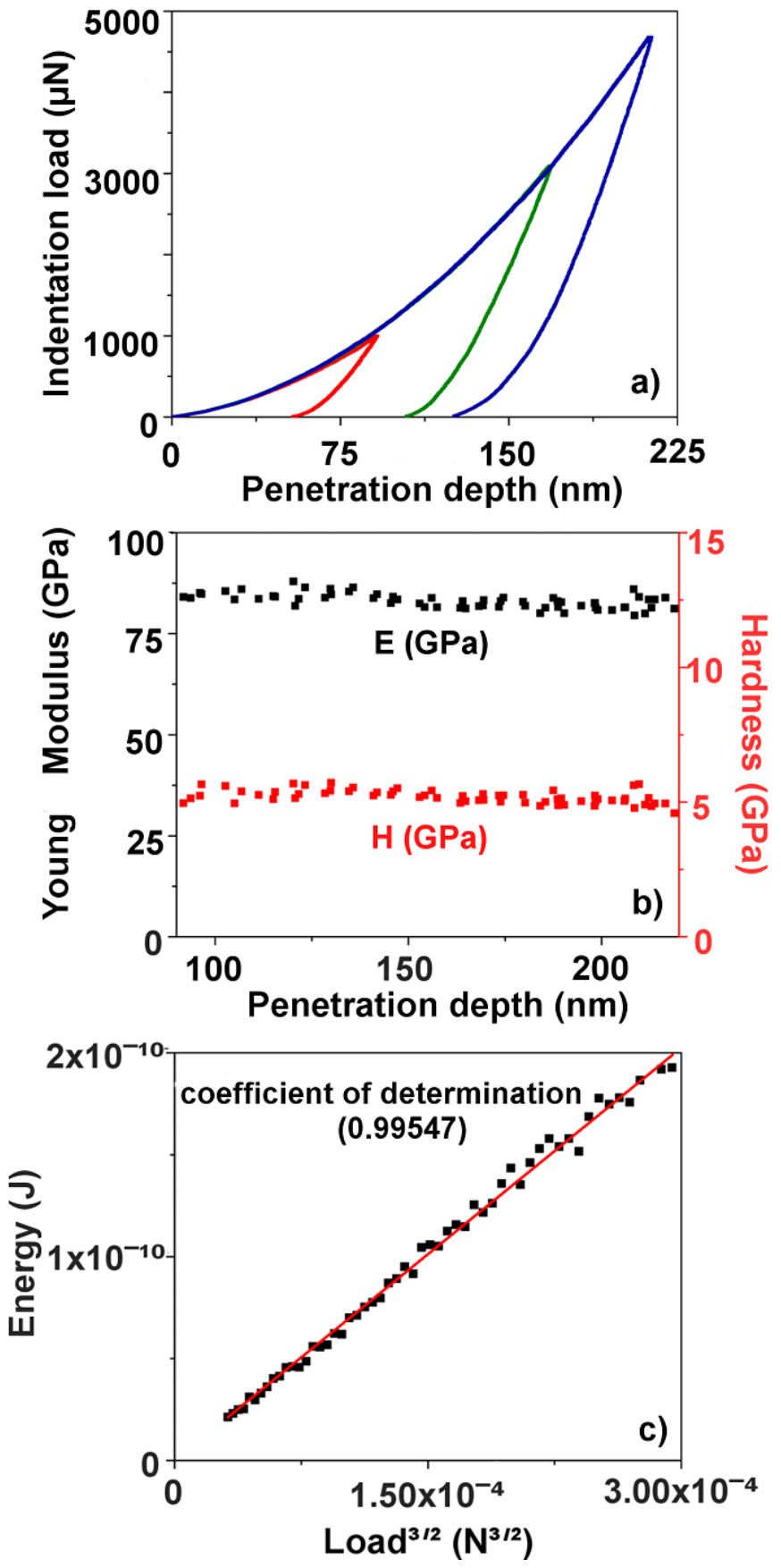

Fig. 1. The nanoindentation results recorded with the sharp Berkovich tip. The $P-h$ curves were registered for different maximum loads of 1000, 3000, and $4700 \mu \mathrm{N}$ (a), which allowed us to obtain the elastic modulus and hardness depth profiles (b). The considerations based on energy principle of indentation (EPI) enabled us to estimate the "true hardness" from the linear relationship between the energy $U_{r}$ consumed for irreversible deformation and $P_{\max }^{3 / 2}$ value (c).
Our further procedure of the collected indentation data (Fig. 1a) led us to estimate the energy $U_{r}$ (see Eq. (2)) consumed for irreversible nanodeformation at different $P_{\max }$ values following Sakai and Nowak $[13,14]$ :

$$
U_{r}=\int_{0}^{h_{p}} A_{p}(h) h^{2} \mathrm{~d} h,
$$

where $A_{p}$ corresponds to the coefficient describing the resistance of a solid to plastic deformation and $h_{p}$ stays for indentation depth limiting the integration interval. The "true hardness" $\left(H_{T}\right)$ of our material was obtained from Eq. (3) describing $U_{r}-P_{\max }^{3 / 2}$ relationship [13, 14]:

$$
U_{r}=\frac{1}{3} \alpha_{0}^{-\frac{1}{2}} \cot (\psi) H_{T}^{-\frac{1}{2}} P_{\max }^{\frac{3}{2}},
$$

where $\alpha_{0}=3 \sqrt{3}, \psi=70.7^{\circ}$ stand for geometry of the indenter (for further details refer to Ref. [15]). The obtained $H_{T}=5.73 \pm 0.04 \mathrm{GPa}$ has a physical sense, in contrast to depth-dependent values of hardness that does not constitute any material constant. Moreover, the selected plot (Fig. 1c) confirms the linear $U_{r}-P_{\max }^{3 / 2}$ relationship of our data with satisfactory fit (coefficient of determination 0.99985) that proves the EPI applies to our MBE-grown GaSb crystal.

Employing the plot of the $P^{2 / 3}-h$ relationship (Fig. 2a) and equation:

$$
P^{\frac{2}{3}}=\left(\frac{4}{3} E_{\mathrm{eff}} R^{\frac{1}{2}}\right)^{\frac{2}{3}} h,
$$

valid for spherical indentation, we detected the exact location of the point indicating the end of elastic deformation that concerns the critical load $P_{c}=1290 \mu \mathrm{N}$. Furthermore, employing relationship between the contact stress $\sigma$, indenter load $P$, contact area $A$, indenter radius $R$, and contact radius $a$ :

$$
\sigma=\frac{P}{A}, \quad A=\pi R h, \quad a=\sqrt{R h}
$$

we were able to calculate the yield strength $\sigma_{Y}=$ $P_{c} / A_{c}=3.8 \mathrm{GPa}\left(A_{c}\right.$ - critical contact area) at the onset of elastic-plastic transition of GaSb crystal (see Fig. 2b).

Our examination of the pop-in that based on spherical indentation data recorded for variety of the maximum loads $P_{\max }$ levels (specify $100-5000 \mu \mathrm{N}$ ) makes us to claim that we determined the moment when the elastic-plastic transition occurs (see Fig. 2a). The detected singularity on the loading part of $P-h$ curves for GaSb may stem from different structural effects such as phase transition to another crystalline structure (similarly to zinc-blende $\rightarrow$ rock-salt transition reported for GaAs [7]) or defects activity. Indeed, similarly to GaAs the phase transition was observed in GaSb at 4.05 GPa [18], and it may involve as well the vacancy nucleation processes [19].

\section{Conclusions}

In sum, we report the results of nanomechanical examination of the MBE-deposited GaSb-crystal thin film. The elastic modulus and hardness of the material in question were determined. The energy of irreversible surface 

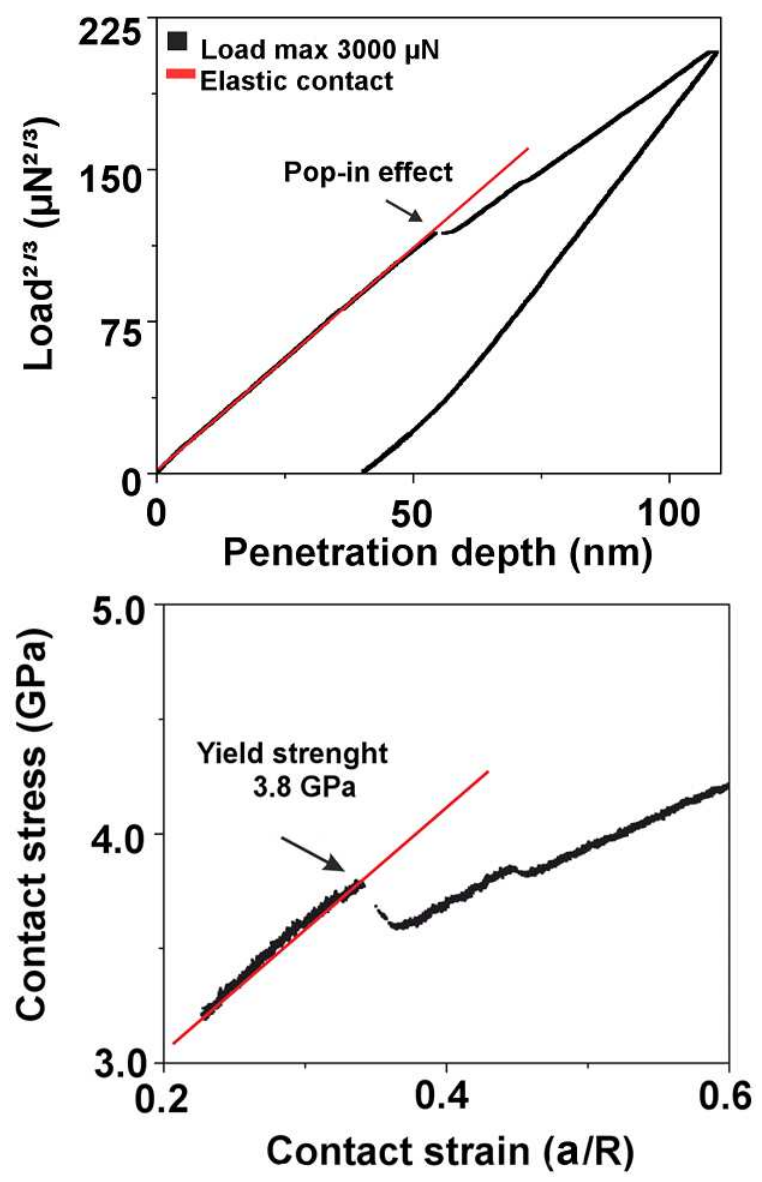

Fig. 2. The results of the spherical indentation in GaSb. The plastic response for the load higher than critical value (pop-in effect) marking the limit of the elastic region of nanodeformed GaSb (a), and provide technologically applicable stress-strain relationship (b).

deformation has been estimated from $P-h$ curves and used to determine the "true hardness" of our material. We found the yield strength of GaSb to be equal to $3.8 \mathrm{GPa}$. Moreover, the spherical indentation revealed the singularity on the load-depth curves (pop-in event) that marks abrupt transition from elastic to plastic behavior. We believe it is caused either by the phase-transitions or by defect activity.

Our results provide a new insight into the mechanical response of the GaSb-crystal, the issue being of interest for fabrication and testing of contemporary semiconducting nanomaterials including GaSb.

\section{Acknowledgments}

A.M., M.T., A.T., D.C., W.B., J.R., and R.N. thank the Academy of Finland for the support through the Research Consortium PROPER within the Finnish Research Program — Programable Materials - OMA. D.C. acknowledges the National Science Center of Poland under grant No. DEC-2012/05/B/ST-8/02945. The authors also wish to thank Mr. Riku Koskinen for preparation of the GaSb samples.

\section{References}

[1] Y. Nagao, T. Hariu, Y. Shibata, IEEE Trans. Electron Dev. 28, 407 (1981).

[2] A.V. Zolotukhin, V.V. Sherstnev, K.S. Savelieva, E. Grebenshchikova, O.Yu. Serebrennikova, N.D. Ilinskaya, S.A. Slobozhanuk, E.V. Ivanov, Y.P. Yakovlev, J. Phys. Conf. Ser. 461, 1 (2013).

[3] A.W. Bett, O.V. Sulima, Semicond. Sci. Technol. 18, 184 (2003).

[4] L. Du, I. Knez, G. Sullivan, R.-R. Du, Phys. Rev. Lett. 114, 096802 (2015).

[5] A.W. Dey, B.M. Borg, B. Ganjipour, M. Ek, K.A. Dick, E. Lind, C. Thelander, L.E. Wernersson, IEEE Electron. Dev. Lett. 34, 211 (2013).

[6] X. Li, T. Ono, Y. Wang, M. Esashi, Appl. Phys. Lett. 83, 3081 (2003).

[7] R. Nowak, D. Chrobak, S. Nagao, D. Vodnick, M. Berg, A. Tukiainen, M. Pessa, Nat. Nanotechnol. 4, 287 (2009).

[8] D. Chrobak, K.-H. Kim, J. Kurzydłowski, R. Nowak, Appl. Phys. Lett. 103, 072101 (2013).

[9] D. Robidas, C. Arunseshan, K.R. Deepthi, D. Arivuolid, Int. J. Mech. Industr. Eng. 3, 2231 (2013).

[10] W.C. Oliver, G.M. Pharr, J. Mater. Res. 7, 1564 (1992).

[11] A.C. Fisher-Cripps, Mechanical Engineering Series. Nanoindentation, 2nd ed., Springer, New York 2004.

[12] J.L. Plaza, E. Diéguez, Phys. Status Solidi 174, 361 (1999).

[13] M. Sakai, R. Nowak, in: Ceramics, Adding the Value, Vol. 2, Ed. M.J. Bannister, Australian Ceramics Society, Melbourne 1992, p. 922.

[14] M. Sakai, Acta Metall. Mater. 41, 1751 (1993).

[15] M. Fujikane, M. Leszczyński, S. Nagao, T. Nakayama, S. Yamanaka, K. Niihara, R. Nowak, J. Alloys Comp. 450, 405 (2008).

[16] K.L. Johnson, Contact Mechanics, 9th ed., Cambridge University Press, Cambridge 2003.

[17] S. Pathak, S.R. Kalidindi, Mater. Sci. Eng. R 91, 1 (2015).

[18] W. Tahar Belarbi, A. Rouabhia, F. Tair, B. Amrani, N. Sekkal, Int. J. Mod. Phys. B 29, 1550056 (2015).

[19] S.G. Corcoran, R.J. Colton, E.T. Lilleodden, W.W. Gerberich, Phys. Rev. B 55, R16057 (1997). 